Submission ID: 43821

\title{
Features of Magnetotelluric Transfer Functions for the Central Part of East
} European Platform

\section{S. Riabova* (Institute Geosphere Dynamics of Russian Academy of Sciences)}

\section{SUMMARY}

Magnetotelluric transfer functions are in demand not only in researching of the Earth's crust geoelectric structure, including in the allocation of the increased fracturing zone, and are also involved in monitoring seismotectonic processes in the lithosphere, establishing effects accompanying earthquakes and searching for possible ore occurrences and oil-gas-saturated deposits. In this work we present an analysis of magnetotelluric transfer functions for the mid-latitude conditions of the East European Platform. The analysis was carried out with the use of instrumental observations made at Geophysical observatory Mikhnevo and Geophysical observatory Borok from 2010 to 2013. The robust method of signal-to-noise separation was used as an evaluation of magnetotelluric transfer functions, which makes it possible to suppress noise of various types. As a result of the analysis of data from observatories Mikhnevo and Borok, the annual periodicity of the variations of the magnetic tipper and the magnetic tensor is established. In the magnetic tensor, annual variations are more pronounced in the variations of the phases of the main components, and in the magnetic tipper variations, along with the annual periodicity, there also are variations with a sixmonth periodicity and with a period of 60 days. 
Особенности магнитотеллурических передаточных функций для центральной части Восточно-Европейской платформы

С.А. Рябова* (Федеральное государственное учреждение науки Институт динамики геосфер Российской академии наук)

\section{Введение}

Магнитотеллурические передаточные функции содержат информацию о геодинамических процессах (Бердичевский и Жданов, 1972). В частности, они востребованы не только при изучении геоэлектрического строения земной коры, в том числе при выделении зоны повышенной трещиноватости, а также привлекаются при мониторинге сейсмотектонических процессов в литосфере, установлении эффектов, сопровождающих землетрясения и при поиске зон возможного рудопроявления и нефтегазонасыщенных месторождений. При магнитотеллурическом анализе используются спектры изменяющихся во времени электрического и магнитного полей на поверхности Земли. Эти поля индуцируются в Земле электромагнитными волнами, распространяющимися в волноводе Земля-ионосфера. Электромагнитные волны создаются естественным образом в результате ионосферных, магнитосферных или атмосферных событий. По измерениям электрического и магнитного полей, поскольку они изменяются со временем на поверхности Земли, можно вычислить магнитотеллурические передаточные функции, такие так: тензор импеданса (Бердичевский, 1960; Тихонов, Бердичевский, 1966), магнитный типпер (Vozoff, 1972), теллурический и магнитный тензоры (Бердичевский и др., 1969).

Впервые результаты исследования временных вариаций магнитотеллурических передаточных функций изложены в статье (Shiraki, 1980). При анализе данных обсерватории Какиока (Бразилия) выявлены сезонные вариации магнитного типпера, которые автор связывает с годовой вариацией электропроводности Земли. Анализ магнитотеллурических передаточных функций позволил выявить суточные, месячные, полугодовые и годовые периодичности, а также 11-летнюю периодичность (Харин 1981; Мороз, Мороз, 2006, 2011; Бабак, Рокитянский, Терешин, 2014).

В настоящей работе исследуются временные вариации магнитотеллурических передаточных функций (магнитный типпер и магнитный тензор) для центральной части ВосточноЕвропейской платформы (Геофизическая обсерватория "Михнево" ИДГ РАН и Геофизическая обсерватория «Борок» ИФЗ РАН).

\section{Использованные данные и методы обработки данных}

В качестве исходных данных использовались результаты инструментальных наблюдений за геомагнитными вариациями на среднеширотной Геофизической обсерватории "Михнево" ИДГ PAH $\left(54,96^{\circ} \mathrm{N} ; 37,77^{\circ} \mathrm{E}\right)$ и на Геофизической обсерватории «Борок» ИФЗ РАН $\left(58,03^{\circ} \mathrm{N}, 38,97\right.$ $\left.{ }^{\circ} \mathrm{E}\right)$, полученные за период 2010-2013 гг.

Геофизическая обсерватория "Михнево" (ГФО МНV) расположена в 85 км к югу от города Москвы, на юге Московской области (неподалеку от города Серпухов). Местоположение обсерватории характеризуется удаленностью от крупных промышленных объектов, что делает ее особо пригодной для изучения динамики магнитотеллурических передаточных функций. Обсерватория "Борок" (ГО ВОХ) расположена в поселке Борок Некоузского района Ярославской области. Обсерватория характеризуется идеальными условиями для проведения электромагнитных измерений - низкий уровень электромагнитных помех, отсутствие промышленных загрязнений. Расстояние между ГФО MHV и ГО ВОХ составляет примерно 270 км. Регистрация на ГФО MHV локальных геомагнитных вариаций выполнялась с 
помощью трехкомпонентного феррозондового магнитометра LEMI-018, а на ГО BOX - с помощью трехосного феррозондового магнитометра IPGP VM391.

Привлекаемые при оценках магнитотеллурических передаточных функций данные инструментальных наблюдений содержат в совокупности результаты воздействия многих источников, интенсивность которых к тому же изменяется во времени. Не все источники информативны при оценке передаточных функций. Например, следует исключать вклад локальных источников естественных и техногенных шумов (Goubau et al., 1978), что вызывает определенные трудности в случае когерентности помехи и полезного сигнала. Спорадическая помеха в используемых данных не может быть выявлена стационарными методами анализа, такими, например, как преобразование Фурье (Chant and Hastie, 1992). Повышение качества оценки магнитотеллурических передаточных функций на конкретном участке земной коры возможно путем привлечения коррелированных временных рядов, полученных на другом участке, для уменьшения или удаления влияния шумовой помехи (Gamble et al., 1978; Chave, Thomson, 2004). Другой путь связан с применением адаптивных робастных методов оценки (Larsen, 1996). В настоящей работе в качестве метода оценки магнитотеллурических передаточных функций использовался робастный метод разделения сигнал/шум (Larsen et al., 1996). Этот метод рассматривался как предпочтительный, так как он позволяет подавлять 1) некоррелированные шумы (аппаратурные), 2) коррелированные (помехи от электрифицированных железных дорог и др.), 3) сигналы локального происхождения (например, ближние молнии), 4) низкий уровень сигнала в "мёртвых" диапазонах.

\section{Результаты}

Анализ данных показал, что магнитные типперы определяются устойчиво на временном интервале длительностью не менее 5 суток. При этом когерентность между вертикальной и горизонтальными составляющими была не менее 0,8 . В качестве примера на рис.1 представлены временные ряды вещественных и мнимых частей магнитного типпера для ГФО MHV за период 2010-2012 г. Данные рис.1 свидетельствуют о наличии периодичностей во временных вариациях магнитного типпера. Наиболее ярко проявляются годовые вариации в поведении модулей индукционных стрелок, что подтверждается спектральной оценкой, результаты которой в виде периодограммы приведены на рис.2. Согласно результатам работы (Мороз и др., 2011) годовые вариации модулей мнимых индукционных стрелок характеризуют вариации электропроводности проводящего слоя, в котором концентрируются электрические токи, вызванные индукцией. Это позволяет предполагать, что выделенная в настоящей работе годовая периодичность связана с наличием мощного проводящего слоя. Представленные на рис.2 периодограммы свидетельствуют о том, что кроме годовой периодичности наблюдаются хорошо выраженные периодичности магнитного типпера с периодами 0,5 года и 60 сут. Годовая и полугодовая периодичности носят сезонный характер. Источник периодичности с 60-ти суточным периодом в настоящее время не ясен и требует проведения дополнительных исследований.

Компоненты магнитного тензора определяются устойчиво на временном интервале длительностью 5 суток. В качестве примера на рис. 3 приведены результаты расчета компонент магнитного тензора. Более детальный анализ свидетельствует о том, что вариации основных компонент и их фаз характеризуются меньшей дисперсией по сравнению с дополнительными компонентами и их фазами. Характерно, что в поведении компонент магнитного тензора выражены годовые вариации, которые по амплитуде в целом превышают дисперсию в 3 - 5 раз. Более четко годовые вариации проявляются в вариациях фаз основных компонент, что, как уже упоминалось, с большой вероятностью связано с изменением электропроводности земной коры. Здесь следует отметить, что поскольку изменение электропроводности земной коры определяется процессами, протекающими в твердых оболочках Земли, выделенные годовые вариации магнитотеллурических передаточных функций на примере магнитного типпера и магнитного тензора могут быть использованы для характеристики геодинамических процессов 


\section{EAGE}

в земной коре, которые характеризуются периодичностями, близкими к 1 году, например, связанными с вращением Земли вокруг Солнца.

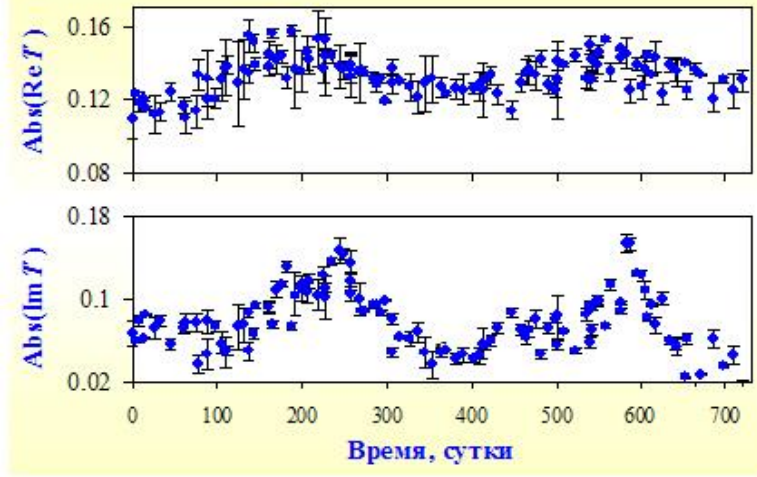

Рис.1. Вариации вещественной (a) и мнимой (б) компонент магнитного типпера на ГФО MHV за период 2010 - 2011 гг.

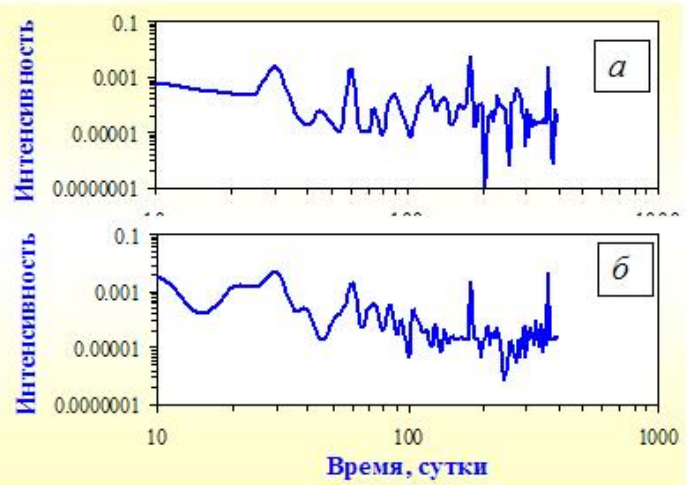

Рис.2. Периодограммы вещественной (а) и мнимой (б) компонент магнитного типпера на ГФО MHV за период 2010 - 2013 гг.

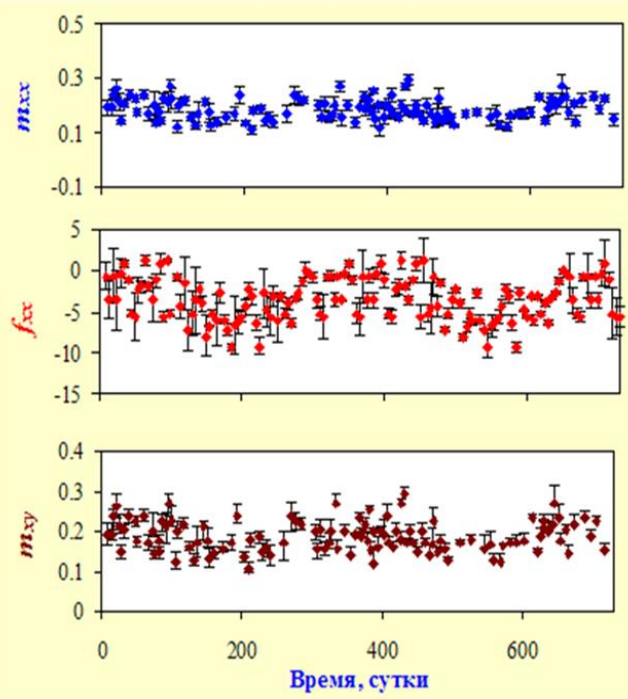

Рис.3. Вариации модуля $\left(m_{x x}\right)$ и фазы $\left(f_{x x}\right)$ основной компоненты и модуля дополнительной компоненты $\left(m_{x y}\right)$ магнитного тензора на ГФО MHV за период 2010 - 2012 гг. 


\section{Выводы}

В результате анализа данных с ГФО «Михнево» и ГО «Борок» установлена годовая периодичность вариаций магнитного типпера и магнитного тензора. При этом в магнитном тензоре годовые вариации в большей мере проявляются в вариациях фаз основных компонент, а в вариациях магнитного типпера наряду с годовой периодичностью наблюдаются также вариации с полугодовой периодичностью и с периодом 60 сут.

\section{Библиография}

Бердичевский М.Н. Основы теории магнитотеллурического профилирования// Прикладная геофизика. 1960. № 28. С. 70-91.

Бердичевский М.Н., Ваньян Л.Л., Файнберг Э.Б. Теоретические принципы использования электромагнитных вариаций для изучения электропроводности Земли// Геомагнетизм и аэрономия. 1969. Т. 9, № 3, С. 570-572.

Мороз Ю.Ф. Смирнов С.Э., Мороз Т.А. Результаты мониторинга вариаций геомагнитного поля на Камчатке// Физика Земли. 2006. № 3. С. 49-56.

Мороз Ю.Ф., Мороз Т.А., Смирнов С.Э. Результаты мониторинга вариаций геомагнитного поля на обсерваториях «Магадан» и «Паратунка»// Физика Земли. 2011. № 8. С. 49-61.

Рокитянский И.И., Бабак В.И., Терешин А.В. К исследованию глубины залегания Карпатской аномалии электропроводности // Геофиз. журн. 2014. Т. 36, № 3. С. 146-159.

Тихонов А.Н., Бердичевский М.Н. Опыт применения магнитотеллурических методов при изучении геологического строения осадочных бассейнов// Физика Земли. 1966. №2, С. 34-41.

Харин Е.П. Изменчивость передаточных функций электромагнитного поля Земли во времени// Геофиз. журнал. 1981. Т.3. № 3. С.16-23.

Chant I.J, Hastie L.M. Time-frequency analysis of magnetotelluric data // Geophys. J. Int.1992. V. 111. No. 2. P. 399-413.

Chave D. J., Thomson A. D. Bounded influence magnetotelluric response function estimation// Geophys. J. Int. 2004. V. 157. No. 3. P. 988-1006.

Gamble T.D., Goubau W.M., Clark J. Magnetotellurics with a remote reference// Geophys. 1979. V. 44. P.53-68.

Goubau W.M., Gamble T.D., Clark J. Magnetotelluric data analysis: removal of bias// Geophys. 1978. V. 43. P.1157-1166.

Larsen I.C., Mackie R.L., Manzella A. Robust smooth magnetotelluric transfer functions// Geophys. J. Int. 1996. V. 124. P. 801-819.

Shiraki M. Monitoring of the time change in transfer functions in the Central Japan conductivity anomaly // J. Geomag. Geoelectr. 1980. V. 32. P. 637-648.

Smirnov M.Yu. Magnetotelluric data processing with a robust statistical procedure having a high breakdown point// Geophys. J. Int. 2003. V. 152. P. 1-7.

Vozoff K. 1972. The magnetotelluric methods in the exploration of sedimentary basins// Geophysics, 37, 98-141. 


\section{References}

Berdichevsky M.N. Principles of Magnetotelluric profiling theory //Journal of Applied Geophysics. 1960. V. 28. P. 70-91.

Berdichevsky M.N., Vanyan L.L., Fainberg E.B., On theoretical principles of using electromagnetic variations for the Earth's conductivity study// Geomagnetism and Aeronomy. 1969. V. 9. P. 570-572.

Chant I.J, Hastie L.M. Time-frequency analysis of magnetotelluric data // Geophys. J. Int.1992. V. 111. No. 2. P. 399-413.

Chave D. J., Thomson A. D. Bounded influence magnetotelluric response function estimation// Geophys. J. Int. 2004. V. 157. No. 3. P. 988-1006.

Gamble T.D., Goubau W.M., Clark J. Magnetotellurics with a remote reference// Geophys. 1979. V. 44. P.53-68.

Goubau W.M., Gamble T.D., Clark J. Magnetotelluric data analysis: removal of bias// Geophys. 1978. V. 43. P.1157-1166.

Kharin E.P. Changes in transfer functions with time// Geophys surveys. 1982. V. 4. No. 4. P. 455466.

Larsen I.C., Mackie R.L., Manzella A. Robust smooth magnetotelluric transfer functions// Geophys. J. Int. 1996. V. 124. P. 801-819.

Moroz Yu.F., Smirnov S.E., Moroz T.A. Results of the monitoring of geomagnetic field variations in the Kamchatka region// Izv. Phys. Earth. 2006. V. 42. No. 3. P. 225-231.

Moroz Yu.F., Moroz T.A.,Smirnov S.E. Results of monitoring the geomagnetic variations at the Magadan and Paratunka observatories // Izv. Phys. Earth. 2011. V. 47. No 8. P. 698-710

Rokityansky I.I., Babak V.I., Tereshin A.V. On the Carpathian electrical conductivity anomaly depth study// Geophysical journal. 2014. V. 36. No. 3. P. 146-159.

Shiraki M. Monitoring of the time change in transfer functions in the Central Japan conductivity anomaly // J. Geomag. Geoelectr. 1980. V. 32. P. 637-648.

Smirnov M.Yu. Magnetotelluric data processing with a robust statistical procedure having a high breakdown point// Geophys. J. Int. 2003. V. 152. P. 1-7.

Tikhonov A.N., Berdichevsky M.N. Experience in the use of magnetotelluric methods to study the geological structures of sedimentary basins// Izv. Phys. Earth. 1966. V. 2. P. 34-41.

Vozoff K. 1972. The magnetotelluric methods in the exploration of sedimentary basins// Geophysics, 37, 98-141. 